Supplementary Information (SI) to

\title{
Hf-Nd-Sr isotopic composition of Tibetan Plateau dust as a fingerprint for regional to hemispherical transport
}

Ting Wei ${ }^{1,3}$, Janice Brahney ${ }^{2}$, Zhiwen Dong ${ }^{1}$, Shichang Kang ${ }^{1,3}$, Chunlei Zong ${ }^{4}$, Junming Guo ${ }^{1}$, Ling Yang ${ }^{1,3}$, and Xiang Qin ${ }^{1,5}$

1 State Key Laboratory of Cryosphere Sciences, Northwest Institute of EcoEnvironment and Resources, Chinese Academy of Sciences, Lanzhou 730000, China;

${ }^{2}$ Department of Watershed Sciences, Utah State University, Logan UT 84322, USA;

${ }^{3}$ University of Chinese Academy of Sciences, Beijing 100049, China;

${ }^{4}$ State Key Laboratory of Continental Dynamics, Northwest University, Xi'an 710069, China;

${ }^{5}$ Qilian Shan Station of Glaciology and Eco-Environment, Chinese Academy of Sciences (CAS), Lanzhou 730000, China.

*Corresponding Author E-mail: dongzhiwen@1zb.ac.cn(Z.Dong)

The SI including 9 Pages, 3 Tables, and 6 Figures. 
Table S1 The annual mean occurrence days of dust storms over the Tibetan Plateau and deserts and sandy lands in China ${ }^{1}$

\begin{tabular}{cccc}
\hline Dust source region & Altitude (m) & $\begin{array}{c}\text { annual mean occurrence } \\
\text { days of dust storms }\end{array}$ \\
\hline western Tibetan Plateau & Shiquanhe & 4279 & 16.5 \\
southern Tibetan Plateau & Lhasa & 3650 & $4 \sim 5$ \\
southern Chang Tang Plateau & Shenzha & 4674 & 16 \\
northeastern Tibetan Plateau & Xinghai & 3324 & 9 \\
Head areas of the Yangtz River & Wudaoliang & 4800 & 12 \\
Taklimakan Desert & & $840-1200$ & 30 \\
Badain Jaran Desert & $1300-1800$ & $20 \sim 30$ \\
Tengger Desert & $1400-1600$ & $20 \sim 30$ \\
Ulan Buh Desert & $1000-1500$ & 20 \\
Gurbantunggut Desert & $200-1000$ & 5 \\
Mu Us sandy land & $1000-1500$ & 10 \\
Hobq sandy land & $1000-2000$ & $10 \sim 20$ \\
Horqin sandy land & $0-500$ & $5 \sim 10$ \\
Otindag sandy land & $1000-1500$ & $5 \sim 10$ \\
Hulun Buir sandy land & $500-1000$ & $1 \sim 1$ \\
\hline
\end{tabular}


Table S2. Mineral composition for the TP dust (weight \%)

\begin{tabular}{|c|c|c|c|c|c|c|c|c|c|c|c|c|c|c|c|c|c|c|c|c|c|c|c|c|c|c|}
\hline Sample No. & $\mathrm{SiO} 2$ & $\mathrm{Al}_{2} \mathrm{O}_{3}$ & $\mathrm{CaO}$ & $\mathrm{Fe}_{2} \mathrm{O}_{3}$ & $\mathrm{MgO}$ & $\mathrm{K}_{2} \mathrm{O}$ & $\begin{array}{l}\mathrm{Na}_{2} \\
\mathrm{O}\end{array}$ & $\mathrm{Cl}$ & $\mathrm{TiO}_{2}$ & $\mathrm{SO}_{3}$ & $\mathrm{P}_{2} \mathrm{O}_{5}$ & $\mathrm{MnO}$ & $\mathrm{SrO}$ & $\mathrm{Cr}_{2} \mathrm{O}_{3}$ & $\mathrm{ZrO}_{2}$ & $\mathrm{~V}_{2} \mathrm{O}_{5}$ & $\mathrm{Rb}_{2} \mathrm{O}$ & $\mathrm{ZnO}$ & $\mathrm{BaO}$ & $\mathrm{NiO}$ & $\mathrm{Co}_{3} \mathrm{O}_{4}$ & $\mathrm{PbO}$ & $\mathrm{CuO}$ & $\mathrm{Ga}_{2} \mathrm{O}_{3}$ & I & $\mathrm{Nb}_{2} \mathrm{O}_{5}$ \\
\hline Q37 & 61.3 & 14.2 & 8.74 & 4.16 & 3.44 & 2.81 & 2.47 & 1.5 & 0.691 & 0.283 & 0.18 & 0.0836 & 0.0371 & 0.0339 & 0.0292 & 0.0149 & 0.0092 & 0.009 & 0.0081 & 0.0038 & 0.0034 & 0.0029 & 0.0026 & 0.0019 & 0.0014 & 0.0011 \\
\hline ТР1803 & 62.5 & 15.98 & 7.37 & 5 & 3 & 2.95 & 1.81 & 0.766 & 0.282 & 0.0974 & 0.0659 & 0.0299 & 0.0253 & 0.0225 & 0.0223 & 0.0217 & 0.0152 & 0.0102 & 0.0058 & 0.0045 & 0.0044 & 0.0038 & 0.0033 & 0.0024 & 0.0018 & 0.0013 \\
\hline TP1816 & 65.46 & 13.54 & 10.55 & 3.3 & 2.63 & 2.25 & 1.1 & 0.561 & 0.189 & 0.103 & 0.0887 & 0.07 & 0.0316 & 0.0293 & 0.0248 & 0.0212 & 0.0108 & 0.0091 & 0.0081 & 0.0063 & 0.0036 & 0.0036 & 0.0035 & 0.0029 & 0.0029 & 0.0021 \\
\hline TP1822 & 67.98 & 17.7 & 4.08 & 3.74 & 1.91 & 1.84 & 1.43 & 0.611 & 0.321 & 0.106 & 0.0985 & 0.0422 & 0.0216 & 0.0181 & 0.0179 & 0.0163 & 0.016 & 0.009 & 0.0089 & 0.0062 & 0.0038 & 0.0032 & 0.0031 & 0.0026 & 0.0023 & 0.002 \\
\hline TP1832 & 67.5 & 16.02 & 4.65 & 3.72 & 2.7 & 2.18 & 1.86 & 0.762 & 0.342 & 0.0896 & 0.0319 & 0.0226 & 0.0203 & 0.0198 & 0.0174 & 0.0127 & 0.0121 & 0.0084 & 0.0079 & 0.0074 & 0.006 & 0.0049 & 0.0035 & 0.0028 & 0.0019 & 0.0016 \\
\hline TP1844 & 62.76 & 18.97 & 5.68 & 3.95 & 3.54 & 2.42 & 1.19 & 0.774 & 0.301 & 0.132 & 0.111 & 0.0231 & 0.0217 & 0.021 & 0.0195 & 0.016 & 0.0134 & 0.01 & 0.0097 & 0.0052 & 0.005 & 0.0041 & 0.0033 & 0.0031 & 0.0028 & 0.0028 \\
\hline TP1845 & 65.96 & 20.64 & 4.99 & 3.81 & 1.23 & 1.12 & 0.93 & 0.875 & 0.199 & 0.0535 & 0.0433 & 0.04 & 0.0194 & 0.0179 & 0.0162 & 0.0128 & 0.0092 & 0.0083 & 0.0062 & 0.0041 & 0.0039 & 0.0028 & 0.0026 & 0.0024 & 0.0022 & 0.0016 \\
\hline ТР1847 & 63.57 & 15.76 & 9.4 & 3.84 & 3.55 & 1.3 & 1.17 & 0.785 & 0.313 & 0.0733 & 0.059 & 0.0396 & 0.0229 & 0.0182 & 0.0164 & 0.0153 & 0.0135 & 0.0114 & 0.0075 & 0.0041 & 0.0036 & 0.0031 & 0.0028 & 0.0027 & 0.0024 & 0.0024 \\
\hline TP1850 & 68.5 & 17.77 & 4.08 & 3.22 & 2.38 & 1.53 & 1.24 & 0.669 & 0.287 & 0.114 & 0.0692 & 0.0235 & 0.0219 & 0.0147 & 0.0138 & 0.0138 & 0.0136 & 0.008 & 0.0052 & 0.004 & 0.0032 & 0.0029 & 0.0028 & 0.0028 & 0.0028 & 0.0025 \\
\hline TP1852 & 75.5 & 14.18 & 3.01 & 2.82 & 1.28 & 1.28 & 0.84 & 0.575 & 0.248 & 0.0743 & 0.0526 & 0.0306 & 0.0151 & 0.0111 & 0.0101 & 0.0101 & 0.0088 & 0.007 & 0.0055 & 0.0033 & 0.0032 & 0.0028 & 0.0027 & 0.0026 & 0.0018 & 0.0017 \\
\hline TP1857 & 58.16 & 15.7 & 12.85 & 4.6 & 3.29 & 2.77 & 1.15 & 0.675 & 0.311 & 0.099 & 0.0927 & 0.0914 & 0.054 & 0.0297 & 0.0295 & 0.0147 & 0.0144 & 0.0113 & 0.0109 & 0.0102 & 0.0068 & 0.0054 & 0.0051 & 0.0035 & 0.0028 & 0.0024 \\
\hline TP1864 & 60.2 & 19.57 & 6.77 & 4.24 & 4.11 & 2.43 & 1.27 & 0.655 & 0.327 & 0.123 & 0.0849 & 0.0481 & 0.0232 & 0.0226 & 0.0215 & 0.0185 & 0.0175 & 0.0128 & 0.0109 & 0.0069 & 0.0046 & 0.0046 & 0.004 & 0.0033 & 0.0025 & 0.0024 \\
\hline ТР1871 & 60.27 & 15.09 & 12.99 & 3.68 & 3.19 & 2.32 & 1.21 & 0.56 & 0.285 & 0.148 & 0.0773 & 0.0321 & 0.0259 & 0.0227 & 0.0199 & 0.0138 & 0.0133 & 0.012 & 0.009 & 0.0056 & 0.0048 & 0.0043 & 0.0031 & 0.003 & 0.0029 & 0.0024 \\
\hline TP1891 & 56.34 & 18.92 & 8.37 & 4.38 & 4.18 & 3.74 & 1.68 & 1.21 & 0.577 & 0.196 & 0.145 & 0.0617 & 0.0503 & 0.0307 & 0.0298 & 0.0175 & 0.0128 & 0.0081 & 0.0074 & 0.0068 & 0.0061 & 0.0057 & 0.0056 & 0.0049 & 0.0046 & 0.0034 \\
\hline TP18101 & 70.53 & 16.94 & 3.87 & 3.27 & 1.41 & 1.27 & 1.17 & 0.785 & 0.334 & 0.136 & 0.0977 & 0.0476 & 0.0211 & 0.021 & 0.0189 & 0.0171 & 0.0114 & 0.0103 & 0.0095 & 0.0068 & 0.0058 & 0.0041 & 0.0038 & 0.0031 & 0.002 & 0.0018 \\
\hline YL & 43.28 & 17.05 & 14.35 & 13.11 & 5.89 & 2.72 & 0.85 & 0.665 & 0.498 & 0.425 & 0.325 & 0.252 & 0.179 & 0.141 & 0.085 & 0.0646 & 0.0235 & 0.0166 & 0.0157 & 0.0151 & 0.0107 & 0.0079 & 0.0074 & 0.0038 & 0.0034 & 0.003 \\
\hline daocheng & 48.48 & 13.43 & 12.88 & 9.31 & 8.4 & 2.6 & 1.39 & 1.32 & 1.04 & 0.458 & 0.329 & 0.0947 & 0.0569 & 0.0438 & 0.041 & 0.037 & 0.0218 & 0.0214 & 0.0122 & 0.0111 & 0.0052 & 0.0043 & 0.0041 & 0.0032 & 0.0027 & 0.0024 \\
\hline Gannan & 65.04 & 16.72 & 5.65 & 4.45 & 2.99 & 2.13 & 1.57 & 0.72 & 0.296 & 0.104 & 0.0915 & 0.0799 & 0.0321 & 0.0223 & 0.0189 & 0.0168 & 0.0123 & 0.011 & 0.007 & 0.0069 & 0.0062 & 0.004 & 0.0038 & 0.0035 & 0.0034 & 0.0025 \\
\hline hailuogou & 57.88 & 16.21 & 6.68 & 6.4 & 4.97 & 3.19 & 2.34 & 0.992 & 0.778 & 0.121 & 0.0957 & 0.0723 & 0.0531 & 0.0525 & 0.0451 & 0.0296 & 0.0215 & 0.0201 & 0.0102 & 0.0083 & 0.0057 & 0.0056 & 0.0054 & 0.0034 & 0.0031 & 0.0029 \\
\hline mahanshan & 64.92 & 17.98 & 5.53 & 3.06 & 3 & 2.58 & 1.5 & 0.793 & 0.265 & 0.109 & 0.0688 & 0.0278 & 0.0209 & 0.0189 & 0.0181 & 0.0179 & 0.017 & 0.0117 & 0.0115 & 0.0058 & 0.005 & 0.0046 & 0.0043 & 0.0034 & 0.0034 & 0.0031 \\
\hline
\end{tabular}


Table S3. Elemental composition for the TP dust (weight \%)

\begin{tabular}{|c|c|c|c|c|c|c|c|c|c|c|c|c|c|c|c|c|c|c|c|c|c|c|c|c|c|c|}
\hline Sample No. & $\mathrm{Si}$ & $\mathrm{Al}$ & $\mathrm{Ca}$ & $\mathrm{Fe}$ & $\mathrm{Mg}$ & K & $\mathrm{Na}$ & $\mathrm{Cl}$ & $\mathrm{Ti}$ & Sx & Px & $\mathrm{Mn}$ & $\mathrm{Sr}$ & $\mathrm{Cr}$ & $\mathrm{Zr}$ & V & $\mathrm{Rb}$ & $\mathrm{Zn}$ & $\mathrm{Ba}$ & $\mathrm{Ni}$ & Co & $\mathrm{Pb}$ & $\mathrm{Cu}$ & $\mathrm{Ga}$ & I & $\mathrm{Nb}$ \\
\hline Q37 & 28.66 & 7.51 & 6.25 & 2.91 & 2.07 & 2.33 & 1.83 & 1.5 & 0.414 & 0.113 & 0.0785 & 0.0647 & 0.0314 & 0.0232 & 0.0216 & 0.0083 & 0.0084 & 0.0072 & 0.0073 & 0.003 & 0.0025 & 0.0027 & 0.0021 & 0.0014 & 0.0014 & 0.00077 \\
\hline ТР1803 & 29.22 & 8.46 & 5.27 & 3.5 & 2.49 & 1.78 & 1.34 & 0.459 & 0.123 & 0.0754 & 0.0264 & 0.0253 & 0.0187 & 0.0154 & 0.0179 & 0.0217 & 0.0085 & 0.0093 & 0.0052 & 0.0033 & 0.0041 & 0.003 & 0.0026 & 0.0018 & 0.0012 & 0.00091 \\
\hline ТР1816 & 30.6 & 7.16 & 7.54 & 2.3 & 2.18 & 1.36 & 0.813 & 0.336 & 0.0825 & 0.103 & 0.0355 & 0.0542 & 0.0216 & 0.0248 & 0.0248 & 0.0157 & 0.0087 & 0.0051 & 0.0074 & 0.0056 & 0.0027 & 0.0028 & 0.0026 & 0.0023 & 0.0023 & 0.0019 \\
\hline ТР1822 & 31.78 & 9.37 & 3.39 & 2.61 & 1.42 & 1.31 & 0.863 & 0.367 & 0.14 & 0.0424 & 0.0763 & 0.0312 & 0.0174 & 0.0166 & 0.0179 & 0.0112 & 0.0135 & 0.005 & 0.0083 & 0.0053 & 0.003 & 0.0025 & 0.0027 & 0.0023 & 0.0017 & 0.0016 \\
\hline ТР1832 & 31.56 & 8.48 & 3.25 & 2.66 & 2.24 & 1.62 & 1.12 & 0.457 & 0.149 & 0.0694 & 0.0236 & 0.0155 & 0.0114 & 0.0167 & 0.014 & 0.0103 & 0.0121 & 0.0077 & 0.0062 & 0.0074 & 0.0051 & 0.0042 & 0.0032 & 0.0021 & 0.0015 & 0.0012 \\
\hline ТР1844 & 29.34 & 10.04 & 3.97 & 2.82 & 2.94 & 1.46 & 0.88 & 0.464 & 0.131 & 0.053 & 0.086 & 0.0231 & 0.0161 & 0.0118 & 0.0157 & 0.0109 & 0.0113 & 0.0081 & 0.0089 & 0.0041 & 0.0037 & 0.0035 & 0.0026 & 0.0029 & 0.0021 & 0.0024 \\
\hline ТP1845 & 30.84 & 10.92 & 3.49 & 3.16 & 0.741 & 0.83 & 0.667 & 0.524 & 0.0869 & 0.0214 & 0.0335 & 0.0296 & 0.0133 & 0.0144 & 0.0091 & 0.0117 & 0.0092 & 0.007 & 0.0053 & 0.0038 & 0.0029 & 0.0022 & 0.002 & 0.0018 & 0.0018 & 0.0011 \\
\hline ТР1847 & 29.72 & 8.34 & 6.72 & 2.69 & 2.95 & 0.965 & 0.707 & 0.47 & 0.136 & 0.0293 & 0.0457 & 0.0293 & 0.0157 & 0.0166 & 0.0139 & 0.0123 & 0.0076 & 0.0114 & 0.0061 & 0.0035 & 0.0032 & 0.0025 & 0.0025 & 0.0021 & 0.0018 & 0.0018 \\
\hline ТР1850 & 32.02 & 9.41 & 2.85 & 2.67 & 1.7 & 0.921 & 0.918 & 0.401 & 0.125 & 0.0884 & 0.0277 & 0.0174 & 0.015 & 0.0082 & 0.0111 & 0.0126 & 0.0115 & 0.008 & 0.0041 & 0.0032 & 0.003 & 0.0021 & 0.0022 & 0.0024 & 0.0025 & 0.0019 \\
\hline TP1852 & 35.3 & 7.51 & 2.5 & 1.97 & 0.771 & 0.952 & 0.605 & 0.345 & 0.108 & 0.0575 & 0.0211 & 0.0209 & 0.0112 & 0.0062 & 0.0101 & 0.0092 & 0.0071 & 0.0059 & 0.0045 & 0.0026 & 0.0027 & 0.0022 & 0.0023 & 0.0024 & 0.0016 & 0.0014 \\
\hline ТР1857 & 27.19 & 8.31 & 9.19 & 3.22 & 2.74 & 1.67 & 0.852 & 0.405 & 0.136 & 0.099 & 0.0371 & 0.0708 & 0.0369 & 0.0251 & 0.0218 & 0.0134 & 0.0113 & 0.0091 & 0.0109 & 0.0057 & 0.0063 & 0.0041 & 0.0046 & 0.0026 & 0.0025 & 0.0019 \\
\hline TP1864 & 28.14 & 10.36 & 4.84 & 2.97 & 3.41 & 1.47 & 0.942 & 0.393 & 0.143 & 0.0494 & 0.0658 & 0.0407 & 0.0172 & 0.0155 & 0.0215 & 0.0169 & 0.0098 & 0.0103 & 0.0089 & 0.0062 & 0.0036 & 0.0039 & 0.0037 & 0.0029 & 0.0019 & 0.0018 \\
\hline ТР1871 & 28.18 & 7.99 & 9.28 & 2.57 & 2.65 & 1.4 & 0.901 & 0.336 & 0.124 & 0.0594 & 0.0599 & 0.0258 & 0.0259 & 0.0192 & 0.0136 & 0.0102 & 0.0074 & 0.011 & 0.0073 & 0.0044 & 0.0041 & 0.0037 & 0.0025 & 0.0022 & 0.0022 & 0.0018 \\
\hline ТР1891 & 26.34 & 10.01 & 5.86 & 2.64 & 2.99 & 3.11 & 1.25 & 0.727 & 0.252 & 0.0786 & 0.112 & 0.0617 & 0.0404 & 0.021 & 0.0167 & 0.0148 & 0.0095 & 0.0066 & 0.0068 & 0.005 & 0.0048 & 0.0049 & 0.0045 & 0.0045 & 0.0039 & 0.0022 \\
\hline ТP18101 & 32.97 & 8.96 & 2.71 & 2.72 & 1.04 & 0.764 & 0.839 & 0.47 & 0.146 & 0.0545 & 0.0757 & 0.0352 & 0.0211 & 0.0144 & 0.0152 & 0.0096 & 0.0104 & 0.0087 & 0.0077 & 0.0058 & 0.0049 & 0.0032 & 0.0028 & 0.0029 & 0.0016 & 0.0012 \\
\hline YL & 20.23 & 11.93 & 8.66 & 6.94 & 4.21 & 1.63 & 0.371 & 0.552 & 0.37 & 0.17 & 0.251 & 0.172 & 0.179 & 0.111 & 0.0683 & 0.0362 & 0.0174 & 0.0133 & 0.0115 & 0.0128 & 0.0087 & 0.0063 & 0.0069 & 0.0033 & 0.0022 & 0.0022 \\
\hline daocheng & 22.66 & 9.39 & 6.82 & 5.61 & 6.01 & 1.56 & 1.03 & 0.576 & 0.862 & 0.355 & 0.132 & 0.0648 & 0.0319 & 0.0344 & 0.0329 & 0.0313 & 0.0218 & 0.0158 & 0.009 & 0.0089 & 0.0042 & 0.003 & 0.0037 & 0.003 & 0.0024 & 0.0018 \\
\hline Gannan & 30.4 & 8.85 & 4.04 & 3.11 & 2.48 & 1.28 & 1.17 & 0.431 & 0.129 & 0.0418 & 0.0915 & 0.0619 & 0.0238 & 0.0153 & 0.016 & 0.0094 & 0.0099 & 0.0101 & 0.0057 & 0.0062 & 0.0049 & 0.0032 & 0.0029 & 0.0026 & 0.0027 & 0.0022 \\
\hline hailuogou & 27.06 & 8.58 & 4.77 & 4.48 & 3 & 2.65 & 1.73 & 0.595 & 0.339 & 0.0937 & 0.0383 & 0.0611 & 0.0363 & 0.0389 & 0.0451 & 0.0166 & 0.0193 & 0.0161 & 0.0093 & 0.0068 & 0.0045 & 0.0048 & 0.004 & 0.0029 & 0.0022 & 0.0023 \\
\hline mahanshan & 30.35 & 9.51 & 3.87 & 2.54 & 2.14 & 1.56 & 1.11 & 0.475 & 0.116 & 0.0845 & 0.0275 & 0.0206 & 0.0143 & 0.0106 & 0.0181 & 0.0144 & 0.0144 & 0.0095 & 0.0105 & 0.0046 & 0.0043 & 0.0039 & 0.0032 & 0.003 & 0.0029 & 0.0023 \\
\hline
\end{tabular}



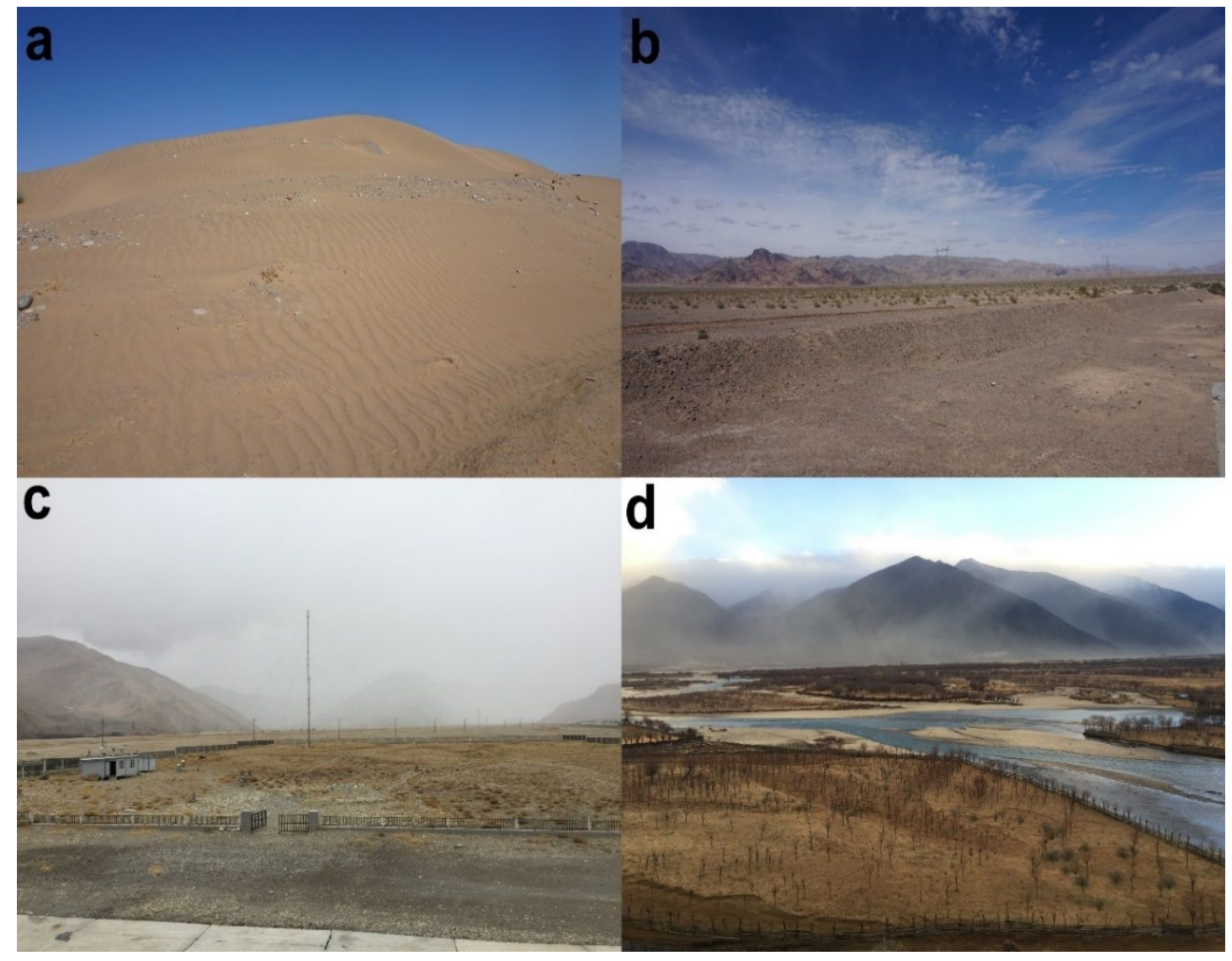

Figure S1 Images of desertification areas and dust storms in the TP. (a) and (b) desert regions in the northern TP; (c) and (d) dust storms in the southern (Everest station) and eastern TP (river valley). 


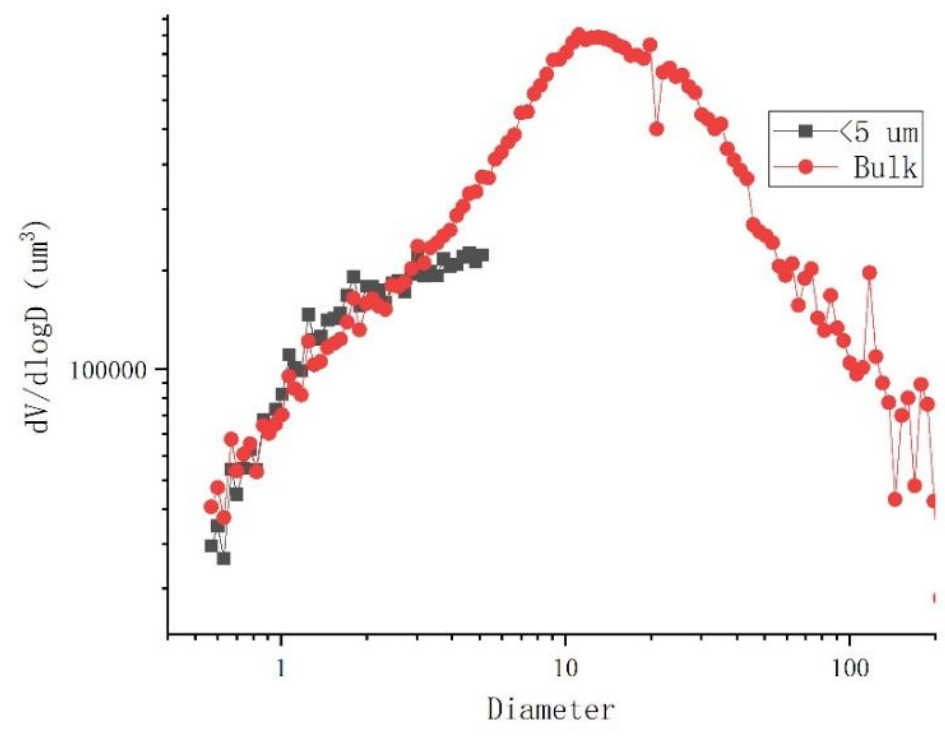

Figure S2 Plot showing the comparison of average mean grain-size distribution of TP surface dust of $<5 \mu \mathrm{m}$ and bulk samples, indicating the TP dust is mainly composed of the lognormal aeolian dust paragraph, and that the grain-size separation worked.

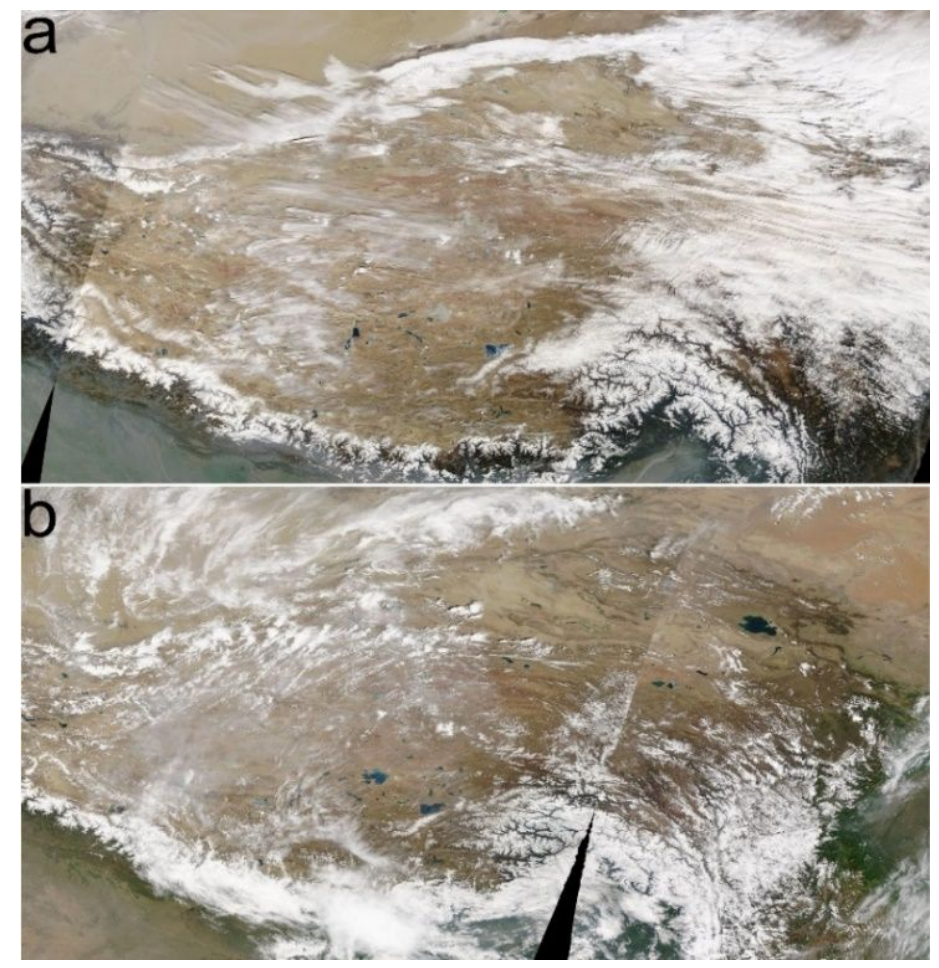

Figure S3 MODIS image (https://worldview.earthdata.nasa.gov/) of dust storm occurrence over the TP in February 20, 2019 (a) and May 14, 2018 (b). 


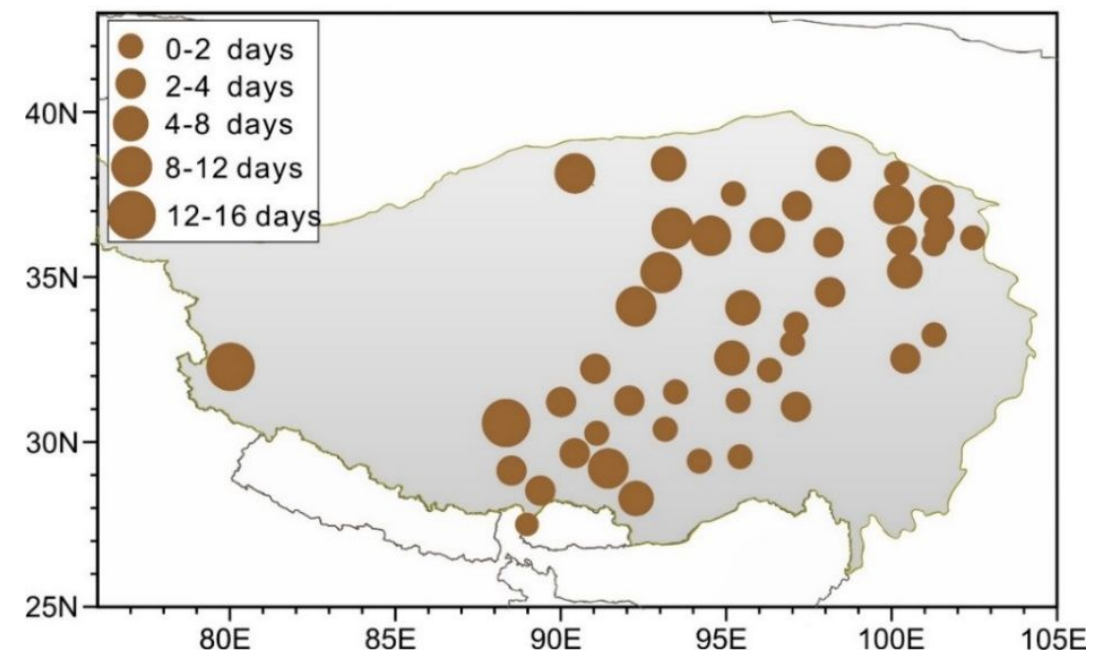

Figure S4 Spatial variation in the annual mean number of dust storm occurrence observed from meteorology stations at the Tibetan Plateau during 1961-2010 (data source: Kang et al. ${ }^{2}$ ).

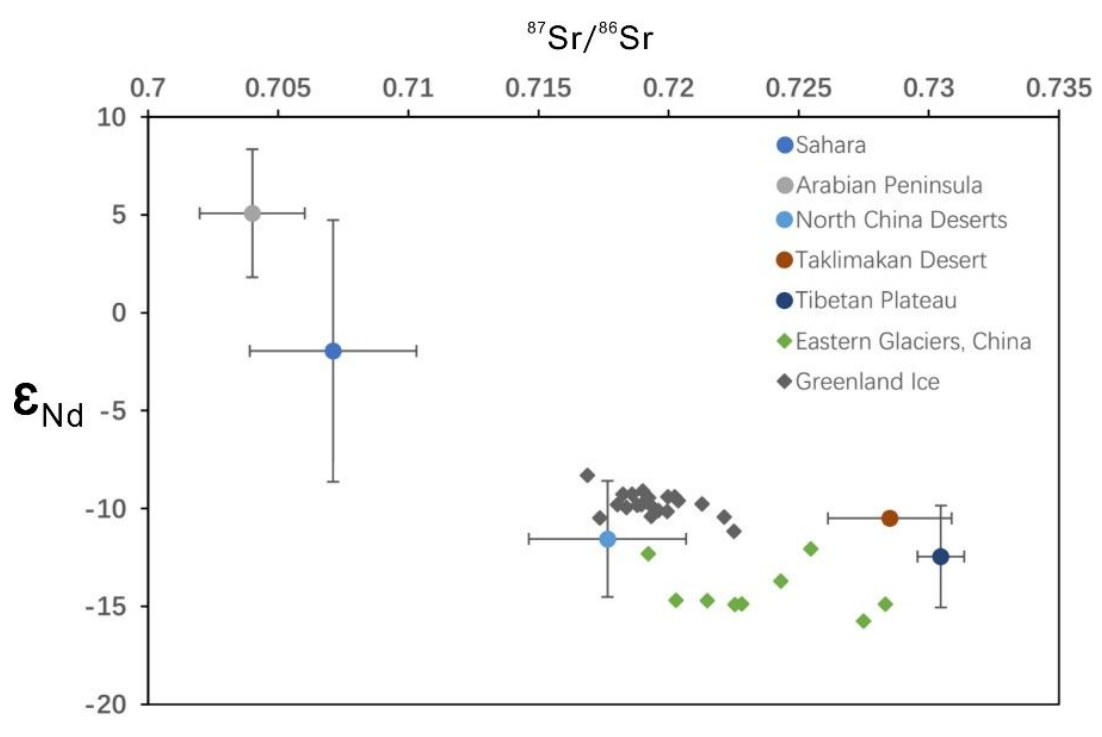

Figure S5. Isotope mixing space for dust sources (circles) and sinks (diamonds). The mean and distribution of each source was used to determine the mean relative contribution to each sink, taking the dust sinks of eastern TP glaciers and Greenland ice as examples. We can find from the figure that, the three major source end members for the sinks (the eastern TP glaciers and Greenland ice) include the Taklimakan Desert, Tibetan Plateau and North China Deserts. 

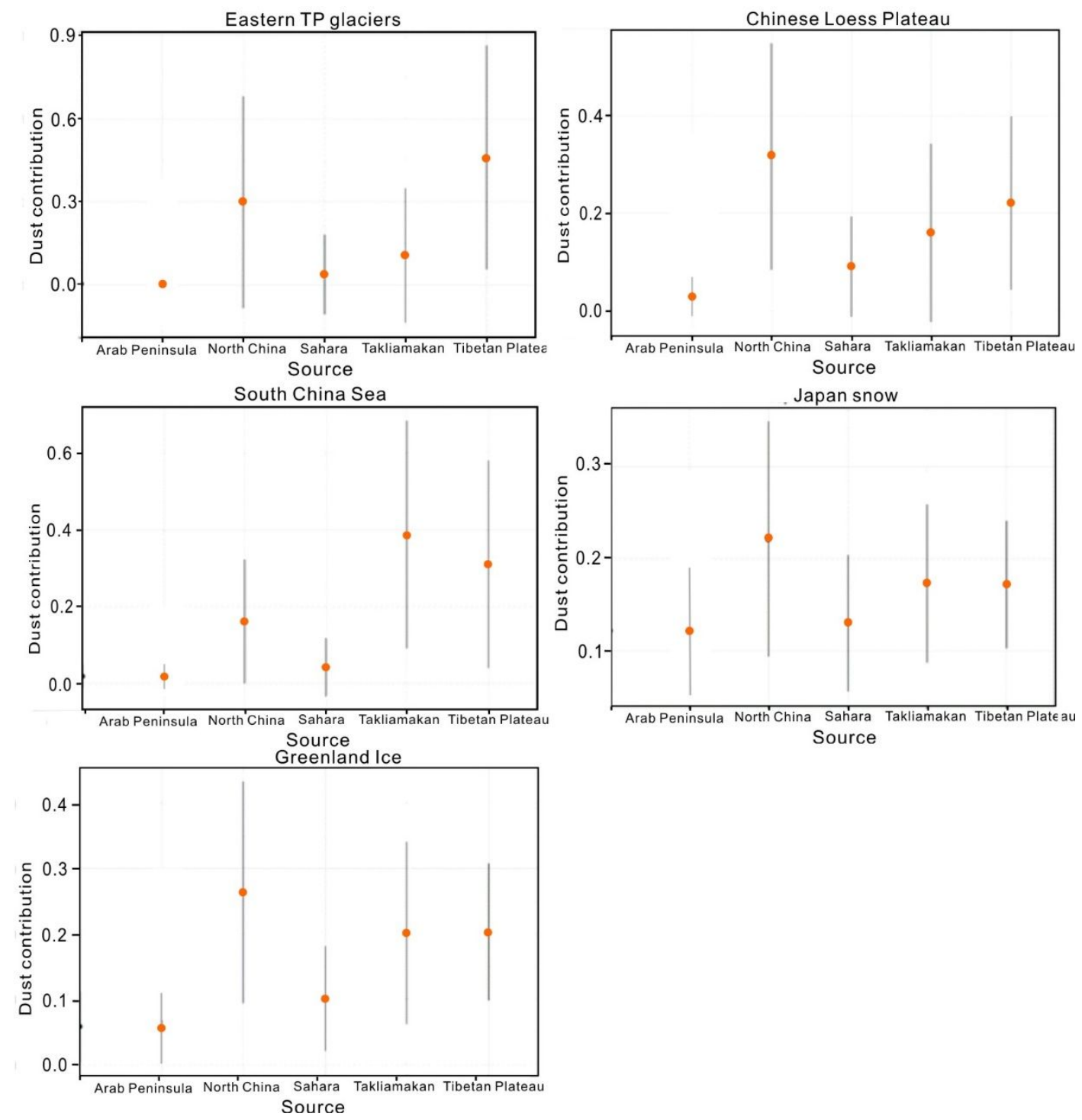

Figure S6. The derived isotope mixing space model for dust sources and sinks. The mean and distribution of each source was used to determine the mean relative contribution to each sink, taking the dust sinks of eastern TP glaciers and Greenland ice as examples. This method is adapted from stream chemistry source separation techniques developed by Christophersen et al. ${ }^{3}$ and Hooper ${ }^{4}$ as well as sediment source separation techniques ${ }^{5}$. The method uses principal components analyses to assign end-member contributions. If the sinks-sediment intervals are bound by all potential end-members, the percent contribution of each end-member to the sediments can be calculated using a constrained least squares equation ${ }^{5}$. Because each end-member is based on several observations, we compute a mean and standard deviation of each end-member.

\section{REFERENCES:}

(1) Fang, X. M.; Han, Y. X.; Ma, J. H.; Song, L. C.; Yang, S. L.; Zhang, X. Y., Dust storms and loess accumulation on the Tibetan Plateau: A case study of dust event on 4 March 2003 in Lhasa. Chinese Science Bulletin 2004, 49, (9), 953-960.

(2) Kang, L.; Huang, J.; Chen, S.; Wang, X., Long-term trends of dust events over Tibetan Plateau 
during 1961-2010. Atmospheric Environment 2016, 125, 188-198.

(3) Christophersen, N.; Neal, C.; Hooper, R. P.; Vogt, R. D.; Andersen, S., Modeling stream water chemistry as a mixture of soil water end - members - A step towards 2nd generation acidification models. Journal of Hydrology 1990, 116, (1-4), 307-320.

(4) Hooper, R. P., Diagnostic tools for mixing models of stream water chemistry. Water Resources Research 2003, 39, (3).

(5) Brahney, J.; Clague, J. J.; Menounos, B.; Edwards, T. W. D., Geochemical reconstruction of late Holocene drainage and mixing in Kluane Lake, Yukon Territory. Journal of Paleolimnology 2008, 40, (1), 489-505. 